ARTICLE

Received 11 Jan 2017 | Accepted 7 Apr 2017 | Published 25 May 2017

DOI: $10.1038 /$ ncomms15586

OPEN

\title{
A scalable double-barcode sequencing platform for characterization of dynamic protein-protein interactions
}

Ulrich Schlecht ${ }^{1,2, \star}$, Zhimin Liu ${ }^{3,4, \star}$, Jamie R. Blundell ${ }^{3,4,5}$, Robert P. St.Onge ${ }^{1,2}$ \& Sasha F. Levy ${ }^{3,4}$

Several large-scale efforts have systematically catalogued protein-protein interactions (PPIs) of a cell in a single environment. However, little is known about how the protein interactome changes across environmental perturbations. Current technologies, which assay one PPI at a time, are too low throughput to make it practical to study protein interactome dynamics. Here, we develop a highly parallel protein-protein interaction sequencing (PPiSeq) platform that uses a novel double barcoding system in conjunction with the dihydrofolate reductase protein-fragment complementation assay in Saccharomyces cerevisiae. PPiSeq detects PPIs at a rate that is on par with current assays and, in contrast with current methods, quantitatively scores PPIs with enough accuracy and sensitivity to detect changes across environments. Both PPI scoring and the bulk of strain construction can be performed with cell pools, making the assay scalable and easily reproduced across environments. PPiSeq is therefore a powerful new tool for large-scale investigations of dynamic PPIs.

\footnotetext{
${ }^{1}$ Stanford Genome Technology Center, Stanford University, 3165 Porter Drive, Palo Alto, Calfornia 94304, USA. ${ }^{2}$ Department of Biochemistry, Stanford University School of Medicine, Stanford, California 94305, USA. ${ }^{3}$ Laufer Center for Physical and Quantitative Biology, Stony Brook University, Stony Brook, New York 11794-5252, USA. ${ }^{4}$ Department of Biochemistry and Cellular Biology, Stony Brook University, Stony Brook, New York 11794-5215, USA.

${ }^{5}$ Department of Applied Physics, Stanford University, Stanford, California 94305, USA. * These authors contributed equally to this work. Correspondence and requests for materials should be addressed to S.F.L. (email: sasha.levy@stonybrook.edu).
} 
A genome-scale understanding of protein-protein interactions (PPIs) has been a long-standing goal of molecular and systems biology ${ }^{1}$. Several large-scale efforts have used the model yeast Saccharomyces cerevisiae to systematically catalogue all PPIs in a single environment using the yeast two-hybrid $(\mathrm{Y} 2 \mathrm{H})$ or the protein fragment complementation assay (PCA) systems ${ }^{2-5}$. These high-throughput screens take advantage of arrayed mating and differential colony growth on agar to identify PPIs. The resulting PPI networks have aided in defining the function of uncharacterized proteins, in characterizing protein complexes and modules that act in concert, and in identifying common functional network motifs ${ }^{2-4,6-9}$. Despite these advances, we are left with a static view of a cell. Unknown is how the protein interactome changes as cells differentiate or react to stress, even though these are each fundamental biological processes ${ }^{10}$. Also missing are PPIs that only occur in non-standard growth conditions.

Describing the global protein interactome dynamics, however, requires overcoming two main hurdles. First, higher throughput is required. S. cerevisiae has $\sim 6,000$ genes, so measuring all pairwise PPIs in one environment requires $\sim 18$ million strains to be assayed, each of which characterizes a single PPI. Observing how the protein interactome changes across perturbations would require re-assaying all $\sim 18$ million strains for each perturbation studied. Even with recent advances such as high density yeast arrays and automated mating and scoring ${ }^{11}$, the time and reagent costs associated with performing screens across several environments make these studies impractical. Recently, a Y2Hand barcode-based pooled assay has been developed, which could potentially greatly increase the throughput of PPI detection ${ }^{12}$. However, because this method relies on plasmid-borne constructs under exogenous promoters, and because $\mathrm{Y} 2 \mathrm{H}$ reporter activation requires nuclear localization, this platform is not ideal for characterizing how PPIs change under natural physiological conditions ${ }^{13}$.

Second, a graded PPI score is necessary to identify PPIs that change. In contrast with barcode-based Y2H, PCA uses genomically integrated fusion proteins under endogenous promoters with protein products interacting in their natural physiological setting, and therefore serves as a viable platform for detecting dynamic PPIs ${ }^{14,15}$. Many different protein fragment designs have been used with PCA, however the highestthroughput version uses a split murine dihydrofolate reductase (mDHFR) that, once reconstructed, allows colony growth on agar containing the $S$. cerevisiae DHFR inhibitor methotrexate (MTX). These assays have historically used colony size thresholds to call whether or not a PPI exists ${ }^{4}$. Graded scoring techniques that assign a fitness to each colony have been employed elsewhere, for example, in synthetic genetic interaction screens ${ }^{16}$. However, it is unclear if these techniques are applicable to $\mathrm{PCA}^{17}$. More recently, we and others have developed methods to detect dynamic PPIs via isolated growth of PCA strains in liquid multiwell plates followed by scoring of optical density growth curves $^{17,18}$, a method that is capable of accurately detecting at least fourfold differences in the number of interacting protein molecules over a broad range of protein abundances ${ }^{17}$. However, the throughput of isolated liquid growth assays is inferior to colony-based assays, making their application to interactomescale studies challenging.

A more scalable alternative is pooled growth of barcoded PCA strains followed by quantification to detect how changes in relative barcode abundance are altered across perturbations ${ }^{18}$. However, this technique is limited in two ways. First, a uniquely barcoded strain must be generated for each PPI, making interactome-scale barcoded libraries impractical to construct (for example, 18 million barcoded strains). Second, changes in relative barcode abundance are detected by comparing to a control condition, as is typical for barcode-based assays ${ }^{12,19-21}$. As we describe here, single time point relative barcode frequencies following growth are sensitive to the fitnesses of other cells in the pool, and therefore do not provide a robust score that can be compared across different pools or environments.

Here, we present a scalable and robust method to identify and quantitatively score dynamic PPIs that we call Protein-Protein interaction Sequencing (PPiSeq). The PPiSeq platform combines PCA, a new genomic double-barcoding technology, time-course barcode sequencing of competing cell pools, and an analytical framework to precisely call fitnesses from barcode lineage trajectories. We use these tools to examine the interactions between $\sim 100$ protein pairs at high replication and across five environments. In a permissive environment, we find that the ability for PPiSeq to identify PPIs is on par with existing assays. In addition, PPiSeq finds that a large fraction of PPIs change across environments, many of which could be validated by other PPI assays. Finally, we show that bulk mating of barcoded strains can generate libraries exceeding $10^{9}$ double barcodes and therefore PPiSeq could potentially be used to simultaneously assay large networks containing thousands of PPIs.

\section{Results}

The PPiSeq platform. We developed a general interaction Sequencing platform (iSeq). Barcodes that are adjacent to a loxP recombination site are introduced at a common chromosomal location in closely related MATa and MAT $\alpha$ haploids. Barcodes are placed on opposite sides of the loxP site in each mating type such that mating and Cre induction causes recombination between homologous chromosomes, resulting in a barcode-loxP-barcode configuration on one chromosome (Fig. 1 and Supplementary Fig. 1). This event is selected for by loxP recombination-induced reassembly of a split URA3 marker ${ }^{22}$. A double barcode unambiguously identifies both parents of a cross in highly complex cell pools, with each barcode half being in close enough proximity to allow the pair to be sequenced together by short-read sequencing. Next, double barcode strains are grown in pools, relative double barcode frequencies are assayed at several times, and their trajectories are used in combination with a global maximum likelihood method to estimate the relative fitness of each strain. Here, we use iSeq in combination with the DHFR PCA system to construct a PPiSeq platform (Fig. 1, Supplementary Fig. 1 and Supplementary Note 1$)^{4}$.

PPiSeq is accurate and highly reproducible. To test the reproducibility of PPiSeq and compare it to existing PPI assays, we selected nine bait (mDHFR-F[1,2]) and nine prey (mDHFR-F[3]) PCA strains and added five different barcodes to each. Bait (IMD3, HOM3, SHR3, PRS3, DBP2, TPO1, DST1, FTR1 and FMP45) and prey (IMD3, FPR1, SHR3, PRS3, DBP2, HXT1, $P D R 5, \quad R P B 9$ and SNQ2) PCA constructs were chosen to encompass a number of previously discovered PPIs. We also added five different barcodes to two control strains that do not contain an mDHFR. Haploid barcoded PCA strains were next pairwise mated and pooled to generate a library of 2,500 double barcode (PPiSeq) strains, with each of the 100 genotypes being represented by 25 unique double barcodes.

We next developed a pooled growth and bar-seq assay capable of robustly measuring the relative fitness of all strains in the pool. We expected that as low fitness PPiSeq strains drop out of the population, the frequency trajectories of a higher fitness strain will begin to 'bend' as its competition gets tougher (green lines, Fig. 2a). The dynamics of this competition depends on the abundances and relative fitnesses of all strains in the pool ${ }^{22}$, and 


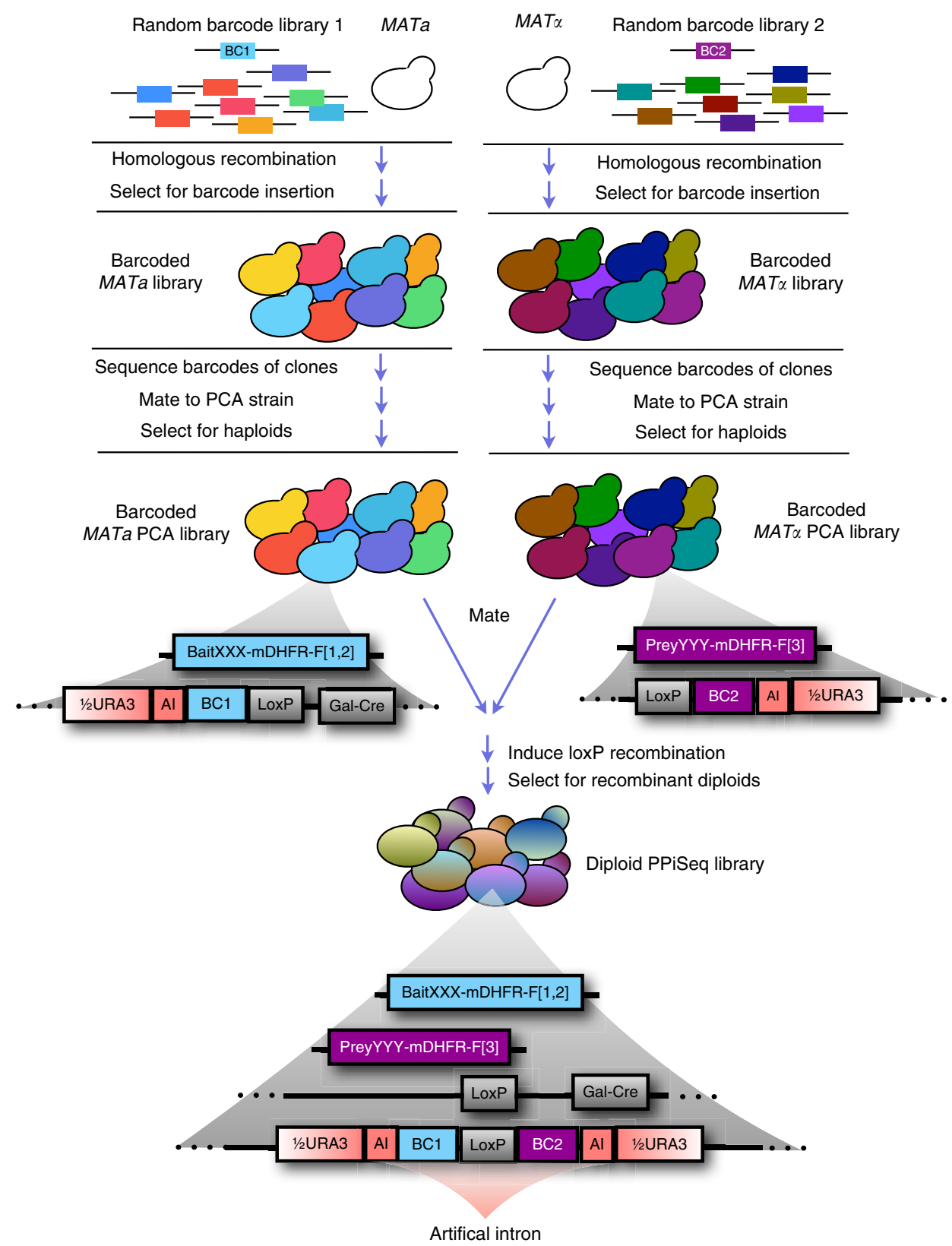

Figure 1 | Construction of a PPiSeq library. Primers containing a random nucleotide barcode are inserted into a common genomic location of both MAT $\alpha$ and MATa cells by homologous recombination, yielding large libraries of barcoded yeast cells. Clones from each library are picked at random and barcodes are identified by sequencing. Barcoded cells are mated to strains containing either a bait or prey protein fragment complementation construct ${ }^{4}$. Diploids are sporulated and haploids containing both a barcode and a PCA construct are selected. These haploids are mated to generate diploids that contain two barcodes and both bait and prey PCA constructs. Cre-induced loxP recombination brings the two barcodes to the same chromosome, and is selected for by reconstruction of a split URA3 selectable marker ${ }^{22}$. The barcode-loxP-barcode sequence resides in an artificial intron and is not translated. Double barcodes mark which two PCA constructs are in each cell and are subsequently used as part of a sequencing-based pooled fitness assay to measure PPI scores.

will therefore change if the composition of the pool changes. Because of this, barcode frequencies at a single time point do not provide a constant measure of fitness across conditions. We therefore monitored relative barcode frequencies over several early time points. We grew the PPiSeq pool in triplicate in standard yeast media and the presence or absence of a low concentration of MTX for $\sim 12$ generations in serial batch culture, diluting 1:8 every $24 \mathrm{~h}(\sim 3$ generations, Supplementary Fig. 2). To allow for fitness measurements of all strains, we chose a low concentration of MTX $\left(0.5 \mu \mathrm{g} \mathrm{ml}^{-1}, 400\right.$-fold lower concentration than traditional $\mathrm{PCA}^{4}$ ) where even strains lacking mDHFR will grow slowly. Double barcodes were sequenced at each dilution (every three generations). We found that reads representing putative PCR chimeras, double barcodes where each double barcode half stems from a different template, occurred at a low but predictable frequency $(\sim 0.2 \%$, Supplementary Note 2) and could confound our results. We therefore subtracted the expected number of PCR chimeras from each double barcode count and generated lineage trajectories with these corrected counts (Fig. 2a and Supplementary Data 1). In the absence of MTX, most PPiSeq strains do not change in frequency over time. However, in the presence of MTX, most strains are driven close to extinction by 12 generations, while others with higher fitness rise in frequency or have a slower decline. Higher 
a
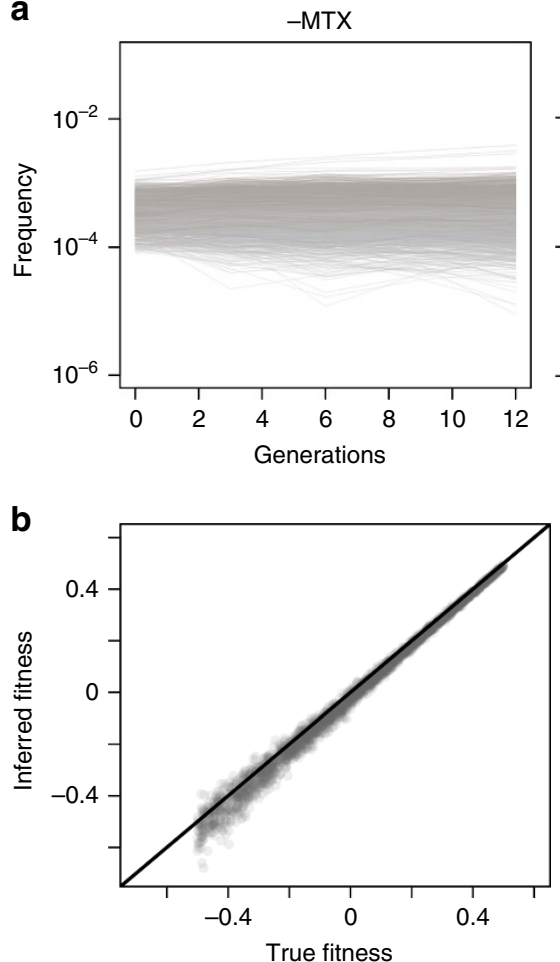

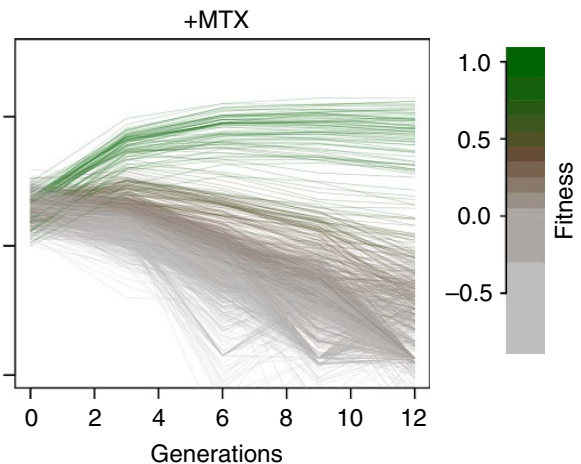

C

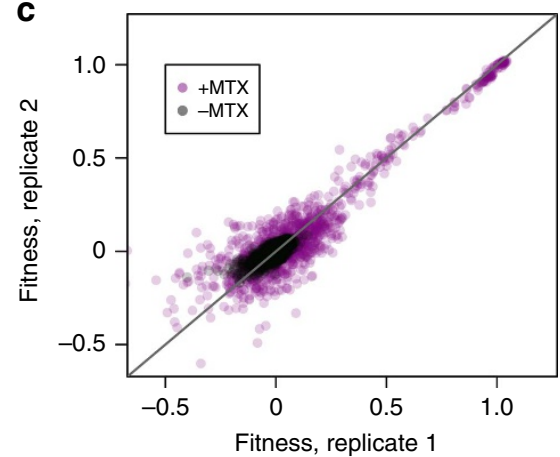

Figure 2 | Lineage tracking and fitness estimation of double barcodes. (a) The frequency trajectories of 2,500 double barcoded PCA strains in the absence or presence of $0.5 \mu \mathrm{g} \mathrm{ml}^{-1}$ methotrexate (MTX). Frequencies are assayed every three generations during serial batch growth. Colour indicates the estimated fitness relative to strains in the same pool that lack mDHFR fragments. (b) Performance of fitness estimates on simulated data. Pearson's $r=0.996$. (c) Reproducibility of fitness estimates across growth replicates. Pearson's $r>0.93$ in MTX.

fitness indicates that protein-mDHFR-fragment pairs within that strain interact to generate complete and functional mDHFR reporter proteins that, in turn, allow the strain to grow faster in the presence of low amounts of MTX.

To robustly calculate the fitness of each trajectory, we use a maximum likelihood strategy under a noise model that accounts for experimental errors (Supplementary Note 3$)^{22}$. To make fitnesses comparable between replicates, or across different barcode pools or environments, we define a strain's fitness relative to the control strain that lacks any mDHFR fragments, whose fitness is set to zero. We find that this procedure performs extremely well on simulated data with parameters similar to our pooled growth experiments (Pearson's $r=0.996$, Fig. $2 \mathrm{~b}$ and Supplementary Note 3 and 4), and across replicate growth experiments (Pearson's $r>0.91$ between all MTX $(+)$ replicates, Fig. 2c). Fitness estimates are generally more accurate for higher fitness strains (those putatively identifying a PPI) because these trajectories are unlikely to fall to low frequencies where counting noise of sequencing reads will be high.

We compared the fitness for each PPI across all $\sim 75$ replicate estimates $(\sim 25$ double barcodes per PPI, 3 replicate growth experiments) in the presence or absence of MTX (Fig. 3 and Methods). Standard errors on fitness are low (typically, s.e.m. $<0.05$ in $\operatorname{MTX}(+)$, Supplementary Fig. 3), with higher fitness PPIs having the lowest errors (s.e.m. $<0.02$ in $\operatorname{MTX}(+)$ for PPIs with fitness $>0.07$ ). We next compared the fitness values of each PPI against the fitness values of the control strains lacking mDHFR in both MTX $(+)$ and MTX $(-)$ conditions (Fig. 3 and Supplementary Data 2). As expected in $\operatorname{MTX}(-)$, almost none of the strains differ significantly in fitness from the control. The single exception is Prs3-F[1,2]:Fpr1-F[3], which displayed a small but highly significant fitness advantage (fitness $=0.04, P$ value $<2 \times 10^{-6}$, Bonferroni corrected one-sided Student's $t$-test) that

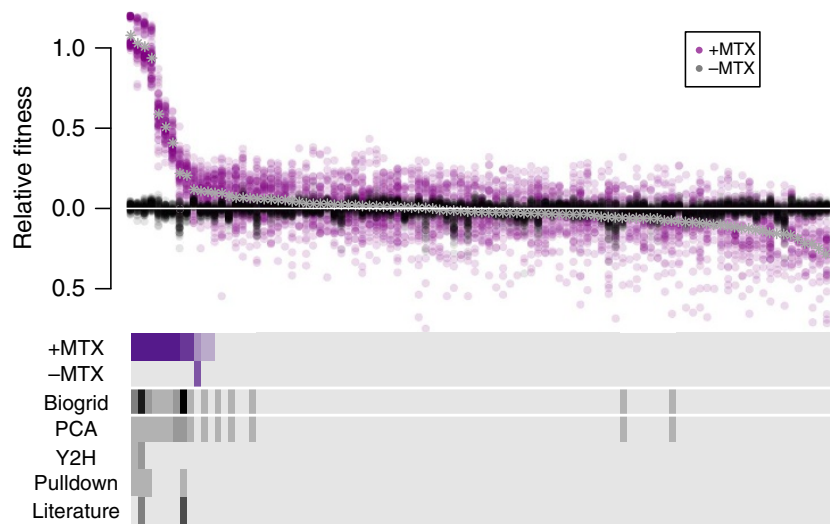

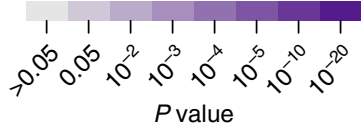

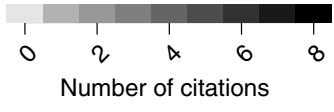

Figure 3 | PPiSeq performance. Top: Relative fitnesses of each protein fragment pair grown in the absence (black) or presence (purple) of MTX. Each protein fragment pair is assayed with 25 unique double barcodes across 3 growth replicates for a total of $\sim 75$ fitness estimates. Asterisks indicate the mean fitness of the protein fragment pair in MTX across all measurements and PPIs are ranked according to this fitness. Bottom purple: Heat map of the significance of the fitness difference between each protein fragment pair and control strains in the same pool that lack mDHFR fragments. $P$ values are calculated using a Bonferroni-corrected Student's $t$-test. Bottom grey: the number of times each protein-protein interaction has previously been cited. Biogrid is the sum of all forms of evidence: protein fragment complementation (PCA), yeast two-hybrid (YTH), pull down/mass spectroscopy (Pulldown), and low-throughput studies (Literature). 
a

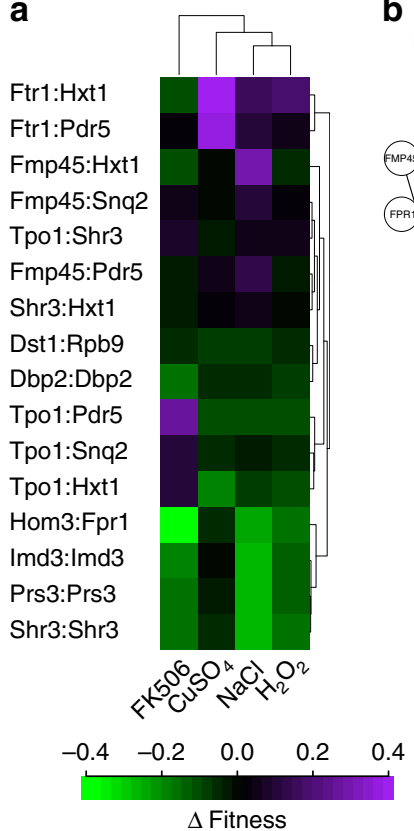

b
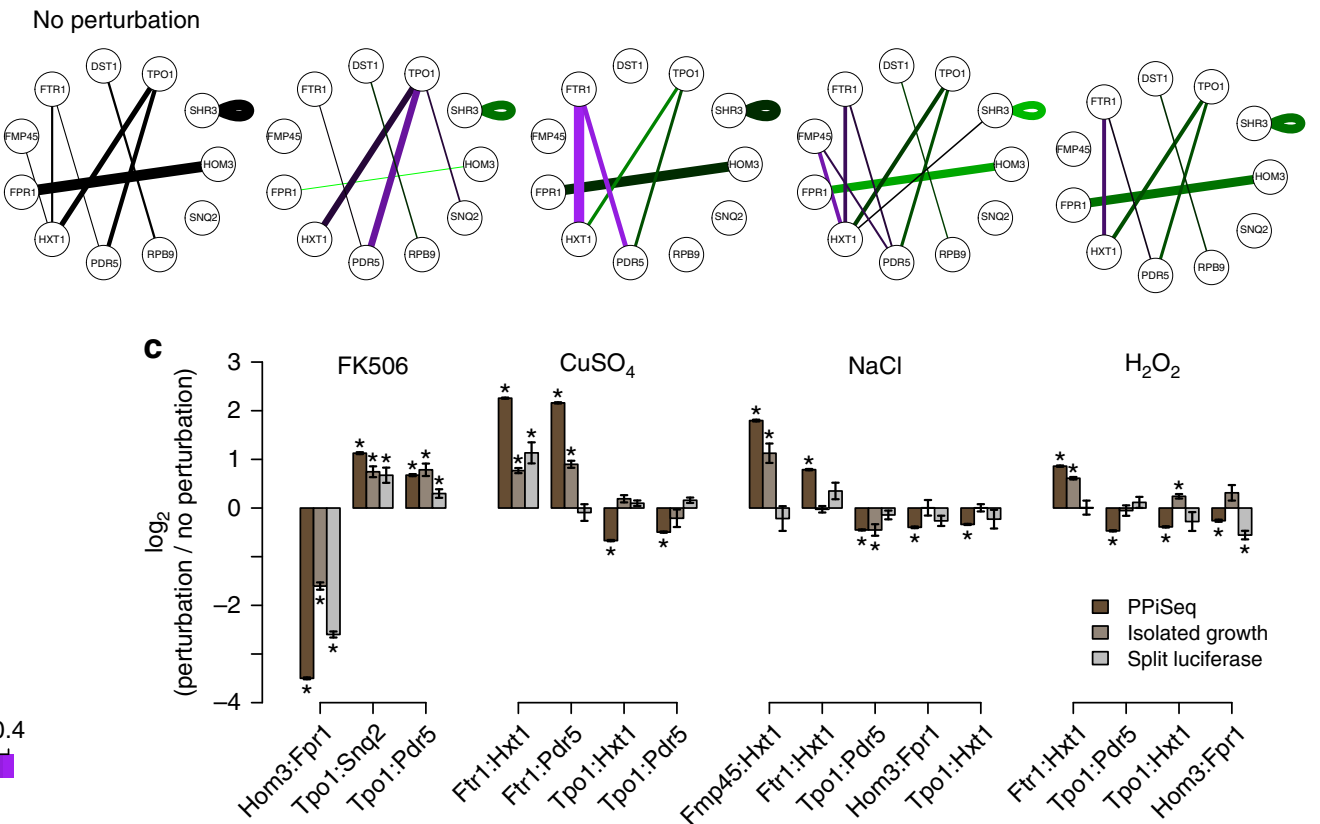

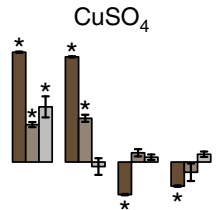

$\left.\right|_{P} ^{*}$
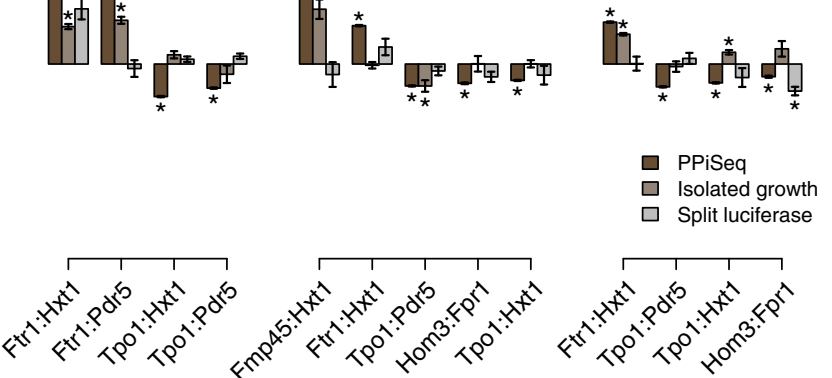

Figure 4 I Dynamic PPIs. (a) Heatmap of PPIs across environments. All PPIs discovered here or elsewhere in standard conditions are shown. Colors are the fitness in each condition minus the fitness in the permissive condition. Cells are arranged by unsupervised hierarchical clustering. (b) PPI network plots of PPIs across five environments. Proteins that only interact with self are omitted. Colours are as in $\mathbf{a}$. Edge width is proportional to the fitness and only significant edges are shown. PPIs expected under the standard condition are listed in $\mathbf{a}$. (c) Barplots of the log ratio of the interaction score of a perturbation over the interaction score in the permissive environment as detected by three assays: PPiSeq (dark brown), split mDHFR clonal growth dynamics (light brown), and split Renilla luciferase luminescence (grey). Error bars are s.e.m. * $p<0.05$, Student's $t$-test against the permissive environment.

is perhaps due to an adaptive mutation that occurred in the parental PCA strain before barcoding. After removing the Prs3:Fpr1 strain from consideration, we find 11 significant PPIs in $\operatorname{MTX}(+), 10$ that have been previously identified ${ }^{4,23}$, and one that is new, Ftr1:Pdr5 (fitness $=0.10, P$ value $<0.002$, Supplementary Data 2). We validated Ftr1:Pdr5 by two additional assays. First, we tracked the optical density (OD600) of Ftr1:Pdr5 PPiSeq strains and the $\operatorname{mDHFR}(-)$ control strains grown in isolation in $\operatorname{MTX}(+)$ media $^{17,18}$ and found that Ftr1:Pdr5 strains rise in optical density faster $(P$ value $<2 \times 10^{-11}$, Student's $t$-test, Supplementary Fig. 4) ${ }^{18}$. Second, we performed a less sensitive Renilla luciferase (Rluc) $\mathrm{PCA}^{24}$ and found that Ftr1:Pdr5 has a consistently higher (but not significant) luminescence when compared to control cells ( $P$ value $=0.24$, Student's $t$-test $)$. As discussed below, the Rluc PCA finds a significant Ftr1:Pdr5 interaction in an alternative environment ( $P$ value $<0.02$ in $200 \mu \mathrm{M}$ copper sulfate, Student's $t$-test), strongly suggesting that our finding in this permissive environment is not a false positive.

Our PPiSeq assay missed five putative PPIs that had been discovered by traditional $\mathrm{PCA}^{4}$. Three (Shr3:Hxt1, Tpo1:Snq2, and Fmp45:Pdr5) showed elevated but not significant fitness increases in $\operatorname{MTX}(+)(0.10,0.08$, and 0.06 , respectively). As discussed below, PPiSeq does find all of these interactions to be significant in at least one perturbation environment, suggesting that these PPIs are sensitive to the environment and that environmental differences between PPiSeq and traditional PCA may impact their detection. The remaining two PPIs (Fmp45:Snq2 and Tpo1:Shr3) could not be detected by PPiSeq in any environment, but could be validated as being PPIs using isolated growth and optical density tracking over $32 \mathrm{~h}$ of growth (Supplementary Fig. 5). Notably, differences in optical density between Tpol:Shr3 and control strains only began to appear around $25 \mathrm{~h}$ of growth, likely caused by a change in Tpol localization following the diauxic shift ${ }^{25,26}$, suggesting that our current $24 \mathrm{~h}$ growth-bottleneck regime is not sensitive to PPIs that are specific to this later growth phase and that longer growth-bottleneck cycles may capture additional PPIs.

Overall, the ability of PPiSeq to detect PPIs appears to be on par with existing PPI assays; in this test set, PPiSeq discovered 10 of 15 PPIs that have been described by other assays, 1 new PPI validated here, and no additional spurious PPIs. When considering other environments, PPiSeq discovered 14 of 16 PPIs that have been discovered here or elsewhere. However, in contrast with previous high-throughput assays, detected PPIs span a reproducible range of positive fitnesses. Growth rate of PCA strains in MTX has previously been found to correlate with the number of functional mDHFR molecules per cell ${ }^{17,18}$, suggesting that fitness differences in our assay are founded in differences in the abundance, localization, or binding of the interacting proteins.

PPiSeq detects dynamic PPIs. One advantage of using a pooled growth and bar-seq approach for detecting PPIs is that, once a barcoded PCA pool is constructed, it is trivial to re-test the entire interaction space across perturbations in order to detect PPIs that are dynamic. Here, we grew the pool of 2,500 PPiSeq strains in triplicate in $\operatorname{MTX}(-)$ and $\operatorname{MTX}(+)$ media supplemented with one of four additional perturbagens: $0.001 \%$ hydrogen peroxide (oxidative stress), $175 \mathrm{mM}$ sodium chloride (high salt), $200 \mu \mathrm{M}$ copper sulfate (high copper), and $50 \mu \mathrm{M}$ of FK506, an inhibitor of calcineurin function in yeast. We calculated the fitness of each strain in each environment relative to the $\operatorname{mDHFR}(-)$ control strain using the maximum likelihood strategy described above. As expected, we found major fitness differences between strains within each $\operatorname{MTX}(+)$ environment, but not within the $\operatorname{MTX}(-)$ environments (Fig. 4, Supplementary Fig. 6, and 
Supplementary Data 4). Surprisingly, $86 \%$ of detected PPIs significantly changed in fitness in at least one perturbation relative to the permissive environment (12 of $14, P<0.05$, Bonferroni corrected Student's $t$-test, Supplementary Data 4$)$ and $50 \%$ were undetectable by our assay in at least one environment (7 of 14, $P>0.05$, Bonferroni corrected one-sided Student's $t$-test, Supplementary Data 5). To validate these changes, we next selected 16 PPI-environment combinations where fitness was significantly different from the permissive environment, and assayed each by both optical density tracking and Rluc PCA (Fig. 4c). We found that 9 of 16 dynamic PPIs could be validated by at least one method.

A number of factors appear to underlie PPI changes across environments. One expected change is the interaction between the aspartate kinase Hom 3 and the peptidyl-prolyl cis-trans isomerase Fpr1 in FK506, which has been previously found to physically disrupt this interaction ${ }^{27}$. Consistent with our previous work $^{18}$, we find that the fitness of the Hom3:Fpr1 PPiSeq strain is diminished $\sim 10$-fold in FK506 $\left(P<10^{-59}\right.$, Supplementary Data 3 and 4). Other dynamic PPIs appear to be due, at least in part, to changes in protein expression. For example, many of the changes that we detect-increased fitness of Tpo1:Pdr5 and Tpo1:Snq2 in FK506, Ftr1:Hxt1 and Ftr1:Pdr5 in copper, and Hxt1:Fmp45 in high salt-co-vary with changes in mRNA expression of one or both interacting partners that are reported in the literature ${ }^{18,28-31}$. Still other dynamic PPIs may be due to changes in protein localization. For example, fitness of the Tpo1:Pdr5 PPiSeq strain decreases in high salt $\left(P<10^{-11}\right)$, even though both TPO1 and PDR5 have been shown to increase in mRNA expression (4.7- and 2.7-fold, respectively $\left.{ }^{32}\right)$. This contradiction appears to be resolved by the finding that Pdr5, but not Tpo1, becomes depleted from the plasma membrane in high salt (Supplementary Fig. 7, Supplementary Note 5) ${ }^{33}$.

PPiSeq is scalable. We have previously shown that at least 500,000 uniquely barcoded strains can be tracked in parallel in a single cell pool ${ }^{22}$. Furthermore, we found that for the majority of barcodes, errors in frequencies are consistent with counting noise stemming from finite read depths, rather than some other factor in the experimental protocol (Supplementary Note 4). Given exponentially declining sequencing costs ${ }^{34}$, it is therefore possible that several million double barcodes could be assayed in parallel. We reasoned that in order for our PPiSeq platform to reach these scales, two criteria must be met. First, PPiSeq must be capable of generating a large number of double barcode strains by pooled mating. Although it is technically possible to probe extremely large interaction spaces by pairwise mating in ordered arrays (for example, ref. 4), the cost and time required to do so is high, and this requirement would greatly reduce the flexibility and scalability of the platform. Second, the distribution of initial double barcode frequencies must be of a form that allows the fitness of most strains in the pool to be measured at reasonable sequencing depths. A distribution where many double barcodes are missing or are present at low frequencies would result in a large fraction of uncharacterized interactions.

To test how many unique double barcodes could be realistically generated by pooled mating, we developed a protocol that mates $\sim 10^{10}$ haploids on a standard agar plate, and then selects for diploid double barcode recombinants (Supplementary Methods). On the basis of experimental tests, we estimated the lower bounds of the frequency of mating $(8 \%)$ and loxP recombination (2\%) of this protocol, and predicted that at least $2 \times 10^{7}$ (i.e. $10^{10} \times 8.1 \% \times 2.7 \%$ ) unique double barcoded diploids are generated per plate (Fig. 5a and Supplementary Note 6). Based on this performance, we estimate that double barcode library sizes
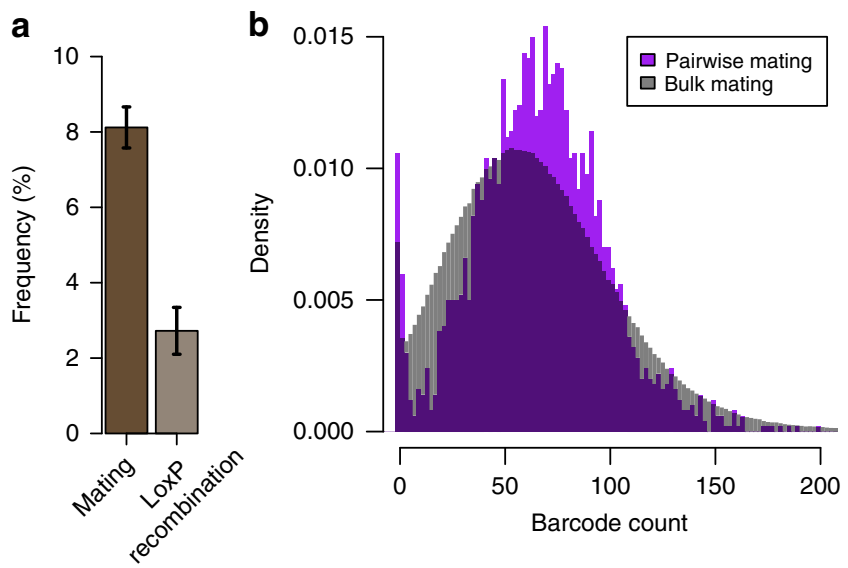

Figure 5 | PPiSeq is scalable. (a) Lower bounds of the mating and loxP recombination efficiencies of a pooled mating and recombination protocol that uses $\sim 10^{10}$ cells per standard plate. Error bars are s.e.m. Each plate has the potential to generate $>2 \times 10^{7}$ double barcodes. (b) Density plot of the frequencies of $\sim 10^{6}$ double barcodes that were generated by bulk mating (grey) and 2,500 double barcodes that were generated by pairwise mating (purple). In both cases, the average number of reads per barcode is 67.

exceeding $10^{9}$ could be achieved by a single investigator $(\sim 50$ mating plates).

We next compared the initial double barcode frequency distribution of a large bulk mating $(\sim 1$ million double barcodes possible across 5 mating plates) to the smaller pairwise mating we used to generate the PPiSeq strains above (2,500 double barcodes possible), and found that the two protocols resulted in similar barcode frequency distributions (Fig. 5b). At an average sequencing depth of $\sim 67$ reads/barcode (Supplementary Note 7), bulk and pairwise mating protocols detect a similar number of double barcodes at low and moderate frequencies ( $>98 \%$ at $>1$ read, $>95 \%$ at $>10$ reads), suggesting that even moderate read depths will be sufficient to characterize most double barcodes in the pool.

\section{Discussion}

We describe a highly parallel PPiSeq assay that is sensitive, accurate, and graded. Importantly, PPiSeq provides a quantitative score (fitness) for each PPI that is robust to changes in the environment or pool constituents. To probe larger interaction sets with PPiSeq, large barcode libraries must first be generated and mated to individual PCA strains. However, we have recently shown that libraries of thousands of barcoded yeast can be generated in parallel using our iSeq platform and Illumina sequencing ${ }^{35}$, and these barcodes can be easily added to PCA strains using the synthetic genetic array technology 11,36 Furthermore, once barcoded PCA libraries have been generated, both double barcode library construction and fitness assays can be performed in large cell pools. PPiSeq is therefore a powerful new platform for large-scale investigations of dynamic PPIs.

The growth of each PCA strain is known to correlate with the number of reconstituted $\mathrm{mDHFR}$ reporter proteins per cell $^{15,17,37}$, which, in turn could be influenced by several factors including the abundance of each interacting protein, the binding affinity, and the extent of co-localization of each binding pair. Protein abundances appear to have a large influence on fitness. For the 16 PPIs in our test set, fitness correlates reasonably well with the abundance of the least abundant interaction partner (Spearman's rho $=0.68$, Supplementary Fig. 8) ${ }^{38}$. Additionally, many of the changes in fitness across environments that we detect 
co-vary with changes in mRNA expression of one or both interacting partners that are reported in the literature. However, other factors are likely to be important as well. For example, a recent proteome-wide screen found that nearly as many proteins change in localization as change in abundance when cells are exposed to hydroxyurea ( 28 and 40 , respectively) ${ }^{39}$. In our test set, we find one example where a change in localization appears to be driving a PPI change (Tpo1:Pdr5). However, we do caution that an unbiased and systematic characterization of the factors underlying dynamic protein interaction will require combining PPiSeq with genome-scale mRNA abundance, protein abundance, and protein localization studies under the same conditions.

For cells treated with FK506, PPiSeq not only detects a change in the PPI target of the drug, Hom3:Fpr1, but also changes in other PPIs such as Tpo1:Snq2 and Tpo1:Pdr5. In this case, additional changes appear to be caused by a specific cellular response to the drug, as each of these proteins are efflux transporters ${ }^{18}$. However, dynamic PPIs that are a response to global changes in the cell physiology or that are due to off-target binding of a drug may also be likely. Avoiding off-target effects, as well as a systems level understanding of a drug's effect on the cell, are often the primary concerns of drug development ${ }^{40-42}$. Because of the ease by which large numbers of PPIs can be quantitatively screened across many perturbations in relatively small volumes of media, PPiSeq therefore provides a powerful new tool for high-throughput drug screening.

More generally, iSeq provides a new framework for performing large-scale dynamic interaction screens. Because PPI scoring and the bulk of strain construction can be performed in cell pools, instead of one-by-one, a major throughput limitation to interaction screens has been removed. Furthermore, iSeq can in theory be used to investigate combinations of any two genetic elements in yeast, such a gene knockouts or engineered constructs, and is therefore likely to have broad utility beyond PPI screens.

\section{Methods}

Pairwise diploid PPiSeq library construction. PPiSeq haploids (Supplementary Note 1 ) were systematically mated to create $50 \times 50=2,500$ diploid strains using standard protocols on a Singer ROTOR HDA robot. Diploid strains were selected on YPD + nourseothricin + hygromycin B. Expression of the Cre-recombinase and strains that successfully recombined their loxP sites were then selected on CSM-uracil + galactose media. A frozen stock of the pool was created by washing the 2,500 strains off the agar plates using YPD $+15 \%$ glycerol and storing aliquots at $-80^{\circ} \mathrm{C}$.
}

Pooled growth assays. An aliquot of the frozen pairwise-mated double barcoded PCA pool was thawed and grown overnight by inoculating $200 \mu \mathrm{l}$ into $20 \mathrm{ml}$ of $\mathrm{YNB}+$ ammonium sulfate + dextrose + histidine + leucine. At late log phase (OD600 = 1.89), four aliquots of $1 \mathrm{ml}$ each were collected, pelleted by centrifugation, and stored as time- 0 samples at $-80^{\circ} \mathrm{C}$. A 48 -well plate was then inoculated with $\mathrm{YNB}+$ ammonium sulfate + dextrose + histidine + leucine media $(700 \mu \mathrm{l})$ with or without $0.5 \mu \mathrm{g} \mathrm{ml}^{-1}$ methotrexate and the pool at a starting OD600 $=0.0525$. The media was supplemented with one of the following components: DMSO (final at 0.5\%), FK506 (final at $50 \mu \mathrm{M}$ ), hydrogen peroxide (final at $0.001 \%$ ), sodium chloride (final at $175 \mathrm{mM}$ ), or copper sulfate (final at $200 \mu \mathrm{M}$ ). Every condition was assayed in triplicate. Every 3 generations (that is, at $3,6,9$ and 12 pool generations), $600 \mu$ l were collected, pelleted by centrifugation and then stored at $-80^{\circ} \mathrm{C} .70 \mu \mathrm{l}$ were inoculated into fresh media of the same type (that is, with or without methotrexate and containing the same component). Genomic DNA was then extracted from all 124 samples using the YeaStar Genomic DNA Kit (Zymo Research), and double barcodes were PCR-amplified using the Q5 High-Fidelity 2X Master Mix (NEB) according to manufacturer instructions. PCR was performed with barcoded up and down sequencing primers (multiplexing tags, see Data File 5) that produce a double index to uniquely identify each sample. PCR products were confirmed by agarose gel electrophoresis. After PCR, samples were combined and bead cleaned with Thermo Scientific Sera-Mag Speed Beads Carboxylate-Modified particles. Sequencing was performed on an Illumina HiSeq 2500 with $25 \%$ PhiX DNA. The PhiX DNA was necessary to increase the read complexity for proper calibration of the instrument (see ref. 22).
Double barcode sequence analysis. Barcode reads were processed with custom written software in Python and R as described ${ }^{22}$, with modifications. Briefly, sequences were parsed to isolate the two barcode regions ( 38 base pairs each), sorted by their multiplexing tags (see above), and removed if they failed to pass any of three quality filters: (1) The average Illumina quality score for both barcode regions must be greater than 30 , (2) the first barcode must match the regular expression $\backslash \mathrm{D}^{*}$ ?(.ACC $\left.|\mathrm{T} . \mathrm{CC}| \mathrm{TA} . \mathrm{C} \mid \mathrm{TAC}.\right) \backslash \mathrm{D}\{4,7\}$ ?AA $\backslash \mathrm{D}\{4,7\}$ ?AA $\backslash \mathrm{D}\{4,7\}$ ?TT $\backslash$ $\mathrm{D}\{4,7\} ?\left(\right.$.TAA $\mid$ A.AA $\mid$ AT.A $\mid$ ATA.) $\backslash \mathrm{D}^{*} \mid \backslash \mathrm{D}^{*}$ ?5' -GTACTAACGGCTAATTTGGTG CC;CA- $3^{\prime} \backslash \mathrm{D}^{* \prime}$, and 3$)$ the second barcode must match the regular expression $\backslash \mathrm{D}^{*}$ ?(.TAT $\left.|\mathrm{T} . \mathrm{AT}| \mathrm{TT} . \mathrm{T} \mid \mathrm{TTA}.\right) \backslash \mathrm{D}\{4,7\}$ ?AA $\backslash \mathrm{D}\{4,7\}$ ?AA $\backslash \mathrm{D}\{4,7\}$ ?TT $\backslash \mathrm{D}\{4,7\}$ ?(.GTA $\mid$ G.TA|GG.A|GGT. $\backslash \mathrm{D}^{\star 1}$. A BLAST database containing all expected double barcodes (76 bases each) was constructed and each read was blasted (word size $=11$, reward $=1$, penalty $=-2$ ) against this database. Double barcode reads that blasted at an $\mathrm{e}<10-28$ ( $\sim 2$ mismatches) to an expected double barcode were summed to calculate as an initial estimate of the read number of each double barcode in each condition.

Comparisons to existing PPI studies. Interaction data was downloaded from the Biogrid on 1 December 2015 (S. cerevisiae version 3.4.131) ${ }^{23,43}$. PPIs we sorted based on the form of evidence: Protein Fragment Complementation (PCA) ${ }^{4,18,44}$ Yeast Two Hybrid (YTH) ${ }^{3,5}$, Affinity Pull-Down Assays (Pulldown) ${ }^{45-48}$, and other lower-throughput methods in the literature (Literature).

Significance test for dynamic PPIs. The fitness of each double barcode strain in each environment was determined as described in Supplementary Note 3. Fitnesses for a given PPI were compared across environments using a two-sided Student's $t$-test Bonferroni corrected for 400 tests.

PPI scoring by isolated growth optical density dynamics. Haploid PCA strains ${ }^{4}$ were streaked from frozen stocks onto YPD to recover isolated colonies. MATa PCA strains harbouring BAIT-DHFR-F[1,2]-NatMX were mated one-by-one to $M A T \alpha$ PREY-DHFR-F[3]-HphMX PCA strains in YPD liquid media. A control diploid strain that lacks DHFR was generated by mating a barcoded $M A T a$ ho::NatMX strain with a barcoded MAT $\alpha$ ho::HphMx strain. Following $12 \mathrm{~h}$ of mating, cells were plated onto YPD + nourseothricin + hygromycin B agar and grown for $48 \mathrm{~h}$ at $30^{\circ} \mathrm{C}$ to select for diploids. One colony of each diploid was inoculated into YPD + nourseothricin + hygromycin B liquid media, grown for $12 \mathrm{~h}$ at $30^{\circ} \mathrm{C}$, and then stored in $15 \%$ glycerol at $-80^{\circ} \mathrm{C}$. Cells were streaked from frozen stocks onto YPD and grown for $48 \mathrm{~h}$ at $30^{\circ} \mathrm{C}$. Three isolated colonies of each strain were suspended in sterilized water and counted. For each replicate, $6.4 \times 10^{4}$ cells were inoculated into $150 \mu \mathrm{l}$ of media in black-walled, clear-bottom 96-well plates (Nunc \#265301). Media was synthetic dextrose supplemented with standard concentrations of the amino acids histidine, leucine, and uracil, plus methotrexate $\left(0.5 \mu \mathrm{g} \mathrm{ml}^{-1}\right)$ and one of the following perturbagens: DMSO (final at 0.5\%), FK506 (final at $50 \mu \mathrm{M}$ ), hydrogen peroxide (final at $0.001 \%$ ), sodium chloride (final at $175 \mathrm{mM}$ ), or copper sulfate (final at $200 \mu \mathrm{M}$ ). Plates were sealed with foil (Costar \# 6570) and shaken at 1,300 r.p.m. (DTS4, Elmi) at $30{ }^{\circ} \mathrm{C}$. The optical density (OD units at $600 \mathrm{~nm}$ ) of each microwell culture was recorded (F500, Tecan) at $0,8,10,12,14,16,18,20,22,24$, and $32 \mathrm{~h}$. The area under the curve (AUC) was calculated as the sum of all OD readings before saturation $(32 \mathrm{~h})$ for each strain in each environment. The relative fitness for a strain in a specific condition was quantified with following equation: $\left(\mathrm{AUC}_{\text {target strain }}-\mathrm{AUC}_{\text {control strain }}\right)_{\text {condition }} /\left(\mathrm{AUC}_{\text {target strain }}-\mathrm{AUC}_{\mathrm{control} \mathrm{strain}}\right)_{\mathrm{DMSO}}$.

Luciferase protein fragment complementation assay. To construct Renilla luciferase (Rluc) PCA strains, we replaced the DHFR fragments with Rluc PCA fragments in haploid DHFR PCA strains ${ }^{4}$ via homologous recombination. The Rluc-F[1]-NatMX homologous recombination cassette was PCR amplified from the pAG25-linker-Rluc-F[1]-NatMX plasmid ${ }^{24}$, and the Rluc-F[2]-HphMX cassette was PCR amplified from the pAG32-linker-Rluc-F[2]-HphMX plasmid ${ }^{24}$ We used the same pair of primers for the amplification of both homologous recombination cassettes. The forward primer ( $5^{\prime}$-GGCGGTGGCGGATCAGGA GGC-3' ${ }^{\prime}$ ) anneals to the linker sequence in pAG25-linker-Rluc-F[1]-NatMX or PAG32-linker-Rluc-F[2]-HphMX. The reverse primer (5'-TTCGACACTGGATG GCGGCGTTAG-3') anneals to the $3^{\prime}$-end of the TEF terminator region of NatMX or HphMX. To increase the recombination efficiency for some genes, it was necessary to add an additional $40 \mathrm{bp}$ to the forward primer that matches genespecific sequence upstream of the stop codon. In all cases, MATa PCA (DHFR$\mathrm{F}[1,2]-\mathrm{NatMX})$ strains were transformed with the Rluc-F[2]-HphMX cassettes and $M A T \alpha$ PCA (DHFR-F[3]-HphMX) strains were transformed with the Rluc-F[1]NatMX cassettes. Transformants were selected by plating on YPD plus the appropriate antibiotic, and proper incorporation of the Rluc PCA cassette was validated by PCR. Next, MATa PCA strains harbouring BAIT-Rluc-F[1]-NatMX were mated one-by-one to MAT $\alpha$ PREY-Rluc-F[2]-HphMX strains in YPD liquid media. Following $12 \mathrm{~h}$ of mating, cells were plated onto YPD + nourseothricin + hygromycin $\mathrm{B}$ agar and grown for $48 \mathrm{~h}$ at $30^{\circ} \mathrm{C}$ to select for diploids. One colony of each diploid was inoculated into YPD + nourseothricin + hygromycin B liquid media, grown for $12 \mathrm{~h}$ at $30^{\circ} \mathrm{C}$, and then stored in $15 \%$ glycerol at $-80^{\circ} \mathrm{C}$. 
Triplicate fresh colonies of each diploid Rluc PCA strain were grown in $5 \mathrm{ml}$ synthetic dextrose media supplemented with standard concentrations of histidine, leucine, and uracil at $30^{\circ} \mathrm{C}$ for $24 \mathrm{~h}$, then diluted $1: 32$ into $5 \mathrm{ml}$ of the same media supplemented DMSO (0.5\%), FK506 $(50 \mu \mathrm{M})$, hydrogen peroxide $(0.001 \%)$, sodium chloride $(175 \mathrm{mM})$, or copper sulfate $(200 \mu \mathrm{M})$. Cells were grown for $24 \mathrm{~h}$ at $30^{\circ} \mathrm{C}$, diluted 1:32 again into fresh media containing the same supplement, and grown for another $6 \mathrm{~h}$. Cells were counted, and $1-2 \times 10^{7}$ cells were pelleted, and resuspended in $180 \mu \mathrm{l}$ phosphate-buffered saline (PBS), pH 7.2 containing $1 \mathrm{mM}$ EDTA. Cells were transferred to white 96-well flat bottom plates (Greiner bio-one \# 655075). The luciferase substrate, benzyl coelenterazine (Nanolight \#301), was diluted 1:10 from the stock $(2 \mathrm{mM}$ in absolute ethanol) using $1 \times \mathrm{PBS}$, and $20 \mu \mathrm{l}$ of diluted substrate was added to each sample (to a final concentration of $20 \mu \mathrm{M}$ ). A Centro LB 960 microplate luminometer (Berthold Technologies) was used to measure the Rluc PCA signal, which was integrated for $10 \mathrm{~s}$. Changes in luminescence in response to a specific condition were calculated by the following equation: luminescence $_{\text {condtion }} /$ luminescence $_{\text {DMSO }}$.

Pooled construction of a large double barcode library. iSeq-barcoded haploid $M A T a$ (1137 SHA345 + BC strains) and MAT $\alpha$ (844 SHA349 + BCs strains) strains were grown to saturation $\left(48 \mathrm{~h}\right.$ at $\left.30^{\circ} \mathrm{C}\right)$ in $100 \mu \mathrm{l} \mathrm{YPD}+\mathrm{G} 418$ in 96 -well plates. Clones of the same mating type were pooled to generate the MATa and MATa barcode pools, and stored in $15 \%$ glycerol aliquots at $-80^{\circ} \mathrm{C}$. The frozen barcode pools were thawed completely at room temperature, and $1.35 \times 10^{9}$ cells of the MAT $\alpha$ pool and $2.9 \times 10^{9}$ cells of MATa pool were each inoculated into $200 \mathrm{ml}$ YPD + G418 and grown for $20 \mathrm{~h}$ at $30^{\circ} \mathrm{C}$. A cell count of each pool was taken, the two pools were combined at equal cell densities, and this mixed pool was streaked onto six YPD plates at a density of $10^{10}$ cells per plate to mate. Cells were grown on YPD for $24 \mathrm{~h}$ at $30^{\circ} \mathrm{C}$, and then all plates were scraped and pooled in water. The number of cells in this pool was counted and $\sim 3.3 \times 10^{10}$ cells ( $\frac{1}{3}$ of all the cells) were plated onto 30 SC-Met-Lys plates at equal cell densities. Cells were incubated for $48 \mathrm{~h}$ at $30^{\circ} \mathrm{C}$ and then replicated onto another 30 SC-Met-Lys plates. After another $48 \mathrm{~h}$ incubation at $30^{\circ} \mathrm{C}$, cells were scraped from the $30 \mathrm{SC}$-Met-Lys plates and pooled in water. All the cells $\left(4.2 \times 10^{10}\right)$ were spun down, resuspended with $1 \mathrm{~L} \mathrm{SC}+\mathrm{Gal}-\mathrm{Ura}$, and grown for $48 \mathrm{~h}$ at $30^{\circ} \mathrm{C}$. Then cells were counted and $100 \mathrm{ml}\left(\sim 8.2 \times 10^{9}\right.$ cells) was inoculated into 1 L SC-Ura media and grown for $48 \mathrm{~h}$ at $30^{\circ} \mathrm{C}$ to further enrich for loxP recombinants. Finally, all the cells were collected to form the pooled diploid barcode library.

Sequencing of bulk mated double barcode pools. Genomic DNA of the pooled diploid barcode library was extracted using the MasterPure Yeast DNA Purification Kit (epicentre \#MPY80200). To completely remove RNA, an extra RNase treatment, DNA precipitation with isopropanol, and washing with $70 \%$ ethanol were added after the recommended protocol from the manufacturer. Double barcode amplicons were generated using a two-step PCR protocol ${ }^{22}$. Briefly, a 5-cycle PCR with OneTaq polymerase (New England Biolabs) was performed in 60 reactions for the large double barcode library, amplifying $\sim 1,000$ copies per unique lineage tag. The PCR products were then pooled and purified with PCR Cleanup columns (Qiagen) at six PCR reactions per column. A second 23-cycle PCR was performed with high-fidelity PrimerSTAR Max plolymerase (Takara) in 30 reactions, with $15 \mu \mathrm{l}$ of cleaned product from the first PCR as template and $50 \mu \mathrm{l}$ total volume per tube. PCR products from all reaction tubes were pooled and purified using a PCR Cleanup column (Qiagen) and eluted into $50 \mu \mathrm{l}$ of water. The appropriate PCR band was isolated by E-Gel agarose gel electrophoresis (Life Technologies) and quantitated by Qubit fluorometry (Life Technologies). Sequencing was performed on an Illumina HiSeq 2500 with 25\% PhiX DNA spike-in. The PhiX DNA was necessary to increase the read complexity for proper calibration of the instrument (see ref. 22)

Code availability. All python scripts used to estimate lineage fitness are available from the corresponding author on reasonable request.

Data availability. All barcode sequences and counts for the PPiSeq assays are available in Supplementary Data 1. Additional data sets generated and/or analysed during the current study are available from the corresponding author on reasonable request.

\section{References}

1. Cusick, M. E., Klitgord, N., Vidal, M. \& Hill, D. E. Interactome: gateway into systems biology. Hum. Mol. Genet. 14, R171-R181 (2005).

2. Ito, T. et al. A comprehensive two-hybrid analysis to explore the yeast protein interactome. Proc. Natl Acad. Sci. USA 98, 4569-4574 (2001).

3. Uetz, P. et al. A comprehensive analysis of protein-protein interactions in Saccharomyces cerevisiae. Nature 403, 623-627 (2000).

4. Tarassov, K. et al. An in vivo map of the yeast protein interactome. Science 320, 1465-1470 (2008).

5. Yu, H. et al. High-quality binary protein interaction map of the yeast interactome network. Science 322, 104-110 (2008).
6. Stelzl, U. et al. A human protein-protein interaction network: a resource for annotating the proteome. Cell 122, 957-968 (2005).

7. Spirin, V. \& Mirny, L. A. Protein complexes and functional modules in molecular networks. Proc. Natl Acad. Sci. USA 100, 12123-12128 (2003).

8. Barabási, A.-L. \& Oltvai, Z. N. Network biology: understanding the cell's functional organization. Nat. Rev. Genet. 5, 101-113 (2004).

9. Alon, U. Network motifs: theory and experimental approaches. Nat. Rev. Genet 8, 450-461 (2007)

10. Ideker, T. \& Krogan, N. J. Differential network biology. Mol. Syst. Biol. 8, 565 (2012).

11. Baryshnikova, A. et al. in: Guide to Yeast Genetics: Functional Genomics, Proteomics, and Other Systems Analysis. chapter 7 470, 145-179 (Elsevier Inc., 2010).

12. Yachie, N. et al. Pooled-matrix protein interaction screens using Barcode Fusion Genetics. Mol. Syst. Biol. 12, 863-863 (2016).

13. Mering, Von, C., Krause, R., Snel, B., Cornell, M. \& Oliver, S. G. Comparative assessment of large-scale data sets of protein-protein interactions. Nature 417, 399-403 (2002).

14. Remy, I. \& Michnick, S. W. Visualization of biochemical networks in living cells. Proc. Natl Acad. Sci. USA 98, 7678-7683 (2001).

15. Michnick, S. W., Ear, P. H., Manderson, E. N., Remy, I. \& Stefan, E. Universal strategies in research and drug discovery based on protein-fragment complementation assays. Nat. Rev. Drug Discov. 6, 569-582 (2007).

16. Baryshnikova, A. et al. Quantitative analysis of fitness and genetic interactions in yeast on a genome scale. Nat. Methods 7, 1017-1024 (2010).

17. Freschi, L., Torres-Quiroz, F., Dubé, A. K. \& Landry, C. R. qPCA: a scalable assay to measure the perturbation of protein-protein interactions in living cells. Mol. Biosyst. 9, 36-43 (2013).

18. Schlecht, U., Miranda, M., Suresh, S., Davis, R. W. \& St Onge, R. P. Multiplex assay for condition-dependent changes in protein-protein interactions. Proc. Natl Acad. Sci. USA 109, 9213-9218 (2012).

19. Smith, A. M. et al. Quantitative phenotyping via deep barcode sequencing. Genome Res. 19, 1836-1842 (2009).

20. Gresham, D. et al. System-level analysis of genes and functions affecting survival during nutrient starvation in Saccharomyces cerevisiae. Genetics 187, 299-317 (2011).

21. Gibney, P. A., Lu, C., Caudy, A. A., Hess, D. C. \& Botstein, D. Yeast metabolic and signaling genes are required for heat-shock survival and have little overlap with the heat-induced genes. Proc. Natl Acad. Sci. USA 110, E4393-E4402 (2013).

22. Levy, S. F. et al. Quantitative evolutionary dynamics using high-resolution lineage tracking. Nature 519, 181-186 (2015).

23. Stark, C. et al. BioGRID: a general repository for interaction datasets. Nucleic Acids Res. 34, D535-D539 (2006).

24. Malleshaiah, M. K., Shahrezaei, V., Swain, P. S. \& Michnick, S. W. The scaffold protein Ste5 directly controls a switch-like mating decision in yeast. Nature 465, 101-105 (2010).

25. Albertsen, M., Bellahn, I., Krämer, R. \& Waffenschmidt, S. Localization and function of the yeast multidrug transporter Tpolp. J. Biol. Chem. 278, $12820-12825$ (2003).

26. Uemura, T., Tachihara, K., Tomitori, H., Kashiwagi, K. \& Igarashi, K. Characteristics of the polyamine transporter TPO1 and regulation of its activity and cellular localization by phosphorylation. J. Biol. Chem. 280, 9646-9652 (2005).

27. Arevalo-Rodriguez, M., Wu, X., Hanes, S. D. \& Heitman, J. Prolyl isomerases in yeast. Front. Biosci. 9, 2420-2446 (2004).

28. Gross, C., Kelleher, M., Iyer, V. R., Brown, P. O. \& Winge, D. R. Identification of the copper regulon in Saccharomyces cerevisiae by DNA microarrays. J. Biol. Chem. 275, 32310-32316 (2000).

29. Yasokawa, D. et al. Mechanisms of copper toxicity in Saccharomyces cerevisiae determined by microarray analysis. Environ. Toxicol. 23, 599-606 (2008).

30. Hirayarna, T., Maeda, T., Saito, H. \& Shinozaki, K. Cloning and characterization of seven cDNAs for hyperosmolarity-responsive (HOR) genes of Saccharomyces cerevisiae. Mol. Gen. Genet. 249, 127-138 (1995).

31. Greatrix, B. W. \& van Vuuren, H. J. J. Expression of the HXT13, HXT15 and HXT17 genes in Saccharomyces cerevisiae and stabilization of the HXT1 gene transcript by sugar-induced osmotic stress. Curr. Genet. 49, 205-217 (2006).

32. Yale, J. \& Bohnert, H. J. Transcript Expression in Saccharomyces cerevisiae at high salinty. J. Biol. Chem. 276, 15996-16007 (2001).

33. Szopinska, A., Degand, H., Hochstenbach, J.-F., Nader, J. \& Morsomme, P. Rapid response of the yeast plasma membrane proteome to salt stress. Mol. Cell Proteomics 10, mcp.M111.009589 (2011).

34. Stephens, Z. D. et al. Big Data: astronomical or genomical? PLoS Biol. 13, e1002195 (2015)

35. Smith, J. D. et al. A method for high-throughput production of sequenceverified DNA libraries and strain collections. Mol. Syst. Biol. 13, 913 (2017). 
36. Tong, A. H. et al. Systematic genetic analysis with ordered arrays of yeast deletion mutants. Science 294, 2364-2368 (2001).

37. Remy, I. \& Michnick, S. W. Clonal selection and in vivo quantitation of protein interactions with protein-fragment complementation assays. Proc. Natl Acad. Sci. USA 96, 5394-5399 (1999).

38. Ghaemmaghami, S., Huh, W. K., Bower, K. \& Howson, R. W. Global analysis of protein expression in yeast. Nature 425, 737-741 (2003).

39. Chong, Y. T. et al. Yeast Proteome Dynamics from Single Cell Imaging and Automated Analysis. Cell 161, 1413-1424 (2015).

40. Chang, R. L., Xie, L., Xie, L., Bourne, P. E. \& Palsson, B. Ø. Drug off-target effects predicted using structural analysis in the context of a metabolic network model. PLoS Comput. Biol. 6, e1000938 (2010).

41. Hellerstein, M. K. Exploiting complexity and the robustness of network architecture for drug discovery. J. Pharmacol. Exp. Ther. 325, 1-9 (2008).

42. Hopkins, A. L. Network pharmacology: the next paradigm in drug discovery. Nat. Chem. Biol. 4, 682-690 (2008).

43. Breitkreutz, B. J. et al. The BioGRID Interaction Database: 2008 update. Nucleic Acids Res. 36, D637-D640 (2008).

44. Miller, J. P. et al. Large-scale identification of yeast integral membrane protein interactions. Proc. Natl Acad. Sci. USA 102, 12123-12128 (2005).

45. Ho, Y. et al. Systematic identification of protein complexes in Saccharomyces cerevisiae by mass spectrometry. Nature 415, 180-183 (2002).

46. Krogan, N. J. et al. High-definition macromolecular composition of yeast RNA-processing complexes. Mol. Cell 13, 225-239 (2004).

47. Krogan, N. et al. Global landscape of protein complexes in the yeast Saccharomyces cerevisiae. Nature 440, 637-643 (2006).

48. Babu, M. et al. Interaction landscape of membrane-protein complexes in Saccharomyces cerevisiae. Nature 489, 585-589 (2012).

\section{Acknowledgements}

We are grateful to Stephen Michnick for providing the pAG25-linker-Rluc F[1]-NatMx and PAG32-linker-Rluc F[2]-HphMX plasmids and for technical advice, to Xianan Liu, Richard Bennett, Gavin Sherlock and Mia Jaffe for technical advice, to Daniel Fisher for advice on the analysis, and to Rolf Sternglanz for suggestions on the manuscript. This work was supported by grants from the US National Institutes of Health (R01HG008354 and U01HL127522 to S.F.L.), The Louis and Beatrice Laufer Center, and the New York State Center for Biotechnology.

\section{Author contributions}

S.F.L. wrote the manuscript. U.S., Z.L., J.R.B. and R.P.S.T. contributed to writing the manuscript. U.S., Z.L. and S.F.L performed experiments. Z.L., J.R.B. and S.F.L. analysed data. S.F.L. conceived of and developed the double barcode platform. S.F.L. and U.S. generated the barcoded PPiSeq strains. U.S. developed and performed the PPiSeq assays. U.S. and Z.L. performed the sequencing. Z.L. and S.F.L. analysed the sequencing data. J.R.B. developed the fitness estimation method. Z.L. developed and performed validation assays. Z.L. performed the scalability assays. S.F.L. and Z.L. performed statistics and visualized the data. R.P.S.T. provided essential insight and advice.

\section{Additional information}

Supplementary Information accompanies this paper at http://www.nature.com/ naturecommunications

Competing interests: The authors declare no competing financial interests.

Reprints and permission information is available online at http://npg.nature.com/ reprintsandpermissions/

How to cite this article: Schlecht, U. et al. A scalable double-barcode sequencing platform for characterization of dynamic protein-protein interactions. Nat. Commun. 8, 15586 doi: $10.1038 /$ ncomms15586 (2017).

Publisher's note: Springer Nature remains neutral with regard to jurisdictional claims in published maps and institutional affiliations.

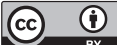

This work is licensed under a Creative Commons Attribution 4.0 International License. The images or other third party material in this article are included in the article's Creative Commons license, unless indicated otherwise in the credit line; if the material is not included under the Creative Commons license, users will need to obtain permission from the license holder to reproduce the material. To view a copy of this license, visit http://creativecommons.org/licenses/by/4.0/

(C) The Author(s) 2017 